\title{
MICROFABRICATED SUBSTRATES FOR THE REMOTE GENERATION OF PRECISION FORCES ON SINGLE CELLS VIA INTRACELLULAR MAGNETIC FLUORESCENT NANOPARTICLES \\ P. Tseng ${ }^{1 *}, J . W . J u d y^{l, 2}$, and D. Di Carlo ${ }^{1,2,3}$ \\ ${ }^{1}$ Electrical Engineering Department \\ ${ }^{2}$ Biomedical Engineering, IDP \\ ${ }^{3}$ Bioengineering Department \\ University of California, Los Angeles, Los Angeles, California, USA
}

\begin{abstract}
A microfabrication process has been investigated and developed for the precision alignment of electroplated ferromagnetic elements with biological cells that are shaped by patterned fibronectin on transparent slides. In addition, we investigate one technique enabled by this process, which is the massively parallel generation of forces on single cells via internalized magnetic-fluorescent nanoparticles. Large magnetic field gradients generated by magnetizing the elements in close proximity to the cells allows the remote generation of large forces on single cells via intracellular localization of nanoparticles, which are capable of polarizing cell shape and activity.
\end{abstract}

\section{INTRODUCTION}

\section{Microfabrication-based force application}

Cellular mechanotransduction, or the method by which cells respond and react to externally and internally generated forces and stresses, plays critical roles in a large number of biological processes, including, but not limited to: mitotic division, cell shape, contractility, polarity, and mobility. Traditionally, researchers attempt to simulate these forces in unwieldy methods, including pipette and AFM tips, however, these lack bulk handling capabilities and tend to significantly perturb cellular systems. ${ }^{1}$

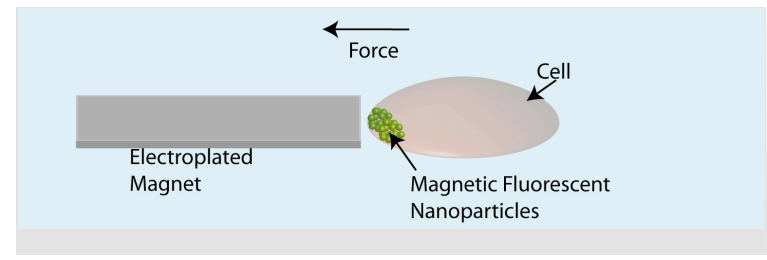

Figure 1: Schematic of the various elements in our system: microfabricated magnets localize and generate forces on magnetic nanoparticles that are internalized within cells.

Magnetic forces have been used to apply simple downward torques and forces on magnetic beads resting above cells, ${ }^{2}$ however this method is limited as the location of the force is entirely random, and these techniques cannot follow moving cells, as well as apply planar forces and shear stresses, which are far more useful in terms effecting cellular response. In an ideal system, large planar forces and shears could be generated and be applied over long periods of time to track cellular response over complicated processes, like through cell division and cell motion. In this work, we combine microfabrication techniques ${ }^{3,4,5}$ and cell-patterning techniques to align force-generating elements in precision proximity to shaped cells. ${ }^{6}$ The cells are loaded with magneticfluorescent nanoparticles, which are then localized within the cell using large fields. ${ }^{7,8,9}$ The small size allows efficient loading and translation of particles within the cells (larger particles are immobilized in the cytoskeletal network), and the limitations on force becomes the particle saturation magnetization and loading densities.

\section{FABRICATION}

Connecting Magnetism and Cells

Our substrates can be separated into three primary components: the magnet layer that focuses external permanent magnetic fields, a planarization layer to generate a flat substrate, and finally surface modification to allow cells to adhere, pattern, and polarize on the substrate.

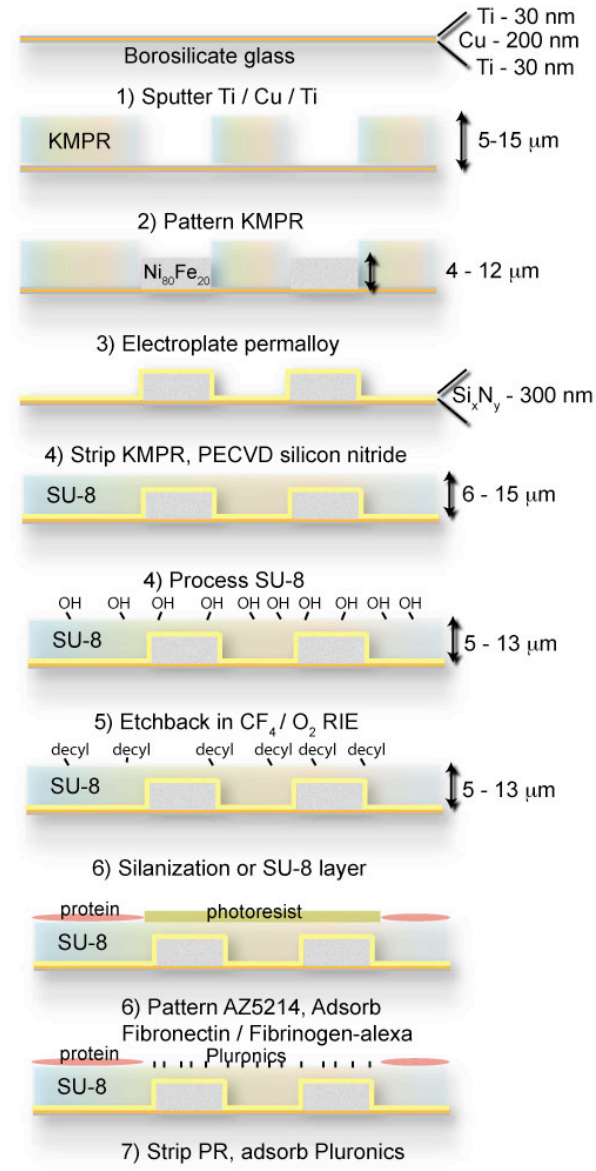

Figure 2: Process flow for our microfabricated, magneto-active slides. Traditional magnetic fabrication and modern cellpatterning techniques are modified to connect cells and magnets for our application. 
Substrate fabrication begins on borosilicate glass (Figure 2). The wafers are cleaned in Pirahna, and evaporated with a $\mathrm{Ti} / \mathrm{Cu} / \mathrm{Ti}$ (30-250-30 nm), and patterned with KMPR photoresist. Permalloy is electrodeposited using a custom-designed setup, and the KMPR is stripped. The thickness of the permalloy plated is dependent to a certain extent on our desired force. Thicker layers have a larger effective reach, and are able to manipulate more particles more efficiently, however are more difficult to planarize. The thickness typically varies from 4 micron to 12 micron in thickness. For the planarization step, SU-8 is spun on, and selectively etched back in $\mathrm{CF}_{4} / \mathrm{O}_{2}$ RIE. The SU-8 surface is then prepared for surface modification.

Cell patterning is adapted from previous methods ${ }^{9}$, and requires a hydrophobic surface to adsorb protein and surfactant. The required surface hydrophobicity of the SU-8 is recovered in two approaches: 1) the additional spinning of a thin layer of SU-8 diluted in PGMEA, or 2) silanization of the oxidized surface in octacdecyl trichlorosilane. AZ5214 resist is then spun onto the substrate, and various patterns are defined in proximity to the elements, including X, I, and square outline patterns. These shapes generate a variety of "square" cell shapes with polarized actin substructure. At this point, these substrates can be stored in this state indefinitely.

In preparation for cell tests, adhesive protein fibronectin is adsorbed, in addition to fibrinogen-alexa fluor conjugates in order to visualize the protein pattern. The photoresist is stripped, and the substrate is immersed in a Pluronics F127 solution to selectively deposit dense polyethylene glycol chains on the surface. Cells can then be patterned onto the substrate.

Magnetic fluorescent nanoparticles are prepared by direct chemical modification of dextran-covered magnetic nanoparticles (nanomag-D, micromod, Germany). The particles are initially cross-linked with the chemical epichlorohydrin, and then subsequently reacted with ammonium hydroxide to generate primary amines on the nanoparticle surface. These are then subsequently conjugated with a selection of fluorophores, depending on the required situation (Texas-Red, RBITC, FITC, alexa-fluor).

\section{SIMULATION}

Magnetic response of the substrate is simulated in both ANSYS and COMSOL (Figure 3). The magnetic element is modeled with our nonlinear ferromagnet $\mathrm{BH}$ loop, and the resultant fields are solved around the element. Extracting the magnetic flux density, we can discern the gradients, and estimate forces on our particles according to the simple equation:

$$
F=\Sigma(m * \nabla B)
$$

where $m$ is the saturation magnetization of an individual nanoparticle, and the summation is over all localized nanoparticles. This equation can be modified to:

$$
F=V * B_{\text {sat }} * \nabla B / \mu_{o},
$$

where $V$ is an estimate of the volume of the nanoparticle cluster.

Depending on the planarization layer thickness, simulations estimate that we are able to achieve magnetic flux density gradients from $1000 \mathrm{~T} / \mathrm{m}$ to $1000000 \mathrm{~T} / \mathrm{m}$ close to the element. We approximate these gradients to generate forces on cells up to and well exceeding those generated during cell motion (10 to $100 \mathrm{pN})$, dependent on the loading density of magnetic nanoparticles inside of cells.

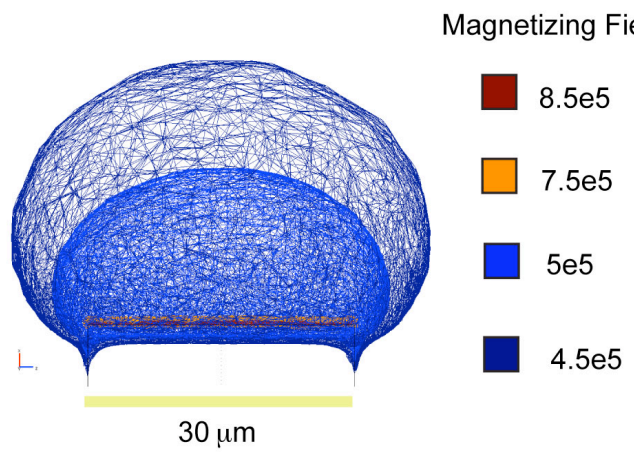

Figure 3: Curves of equal magnetizing fields as simulated in COMSOL. Magnetic particles are localized to areas of large field directly adjacent to the magnetic element.

\section{RESULTS AND DISCUSSION}

\section{Experimental Setup}

Details of our protocol are given elsewhere. In general, cells are seeded onto our substrate, and given 30 minutes to adhere, and then the substrate subsequently washed and inverted above the objective of an inverted fluorescent microscope. Once cells are properly patterned, a magnet is applied to the substrate, and timelapse images are recorded to investigate the response of the cells to the magnetic force.

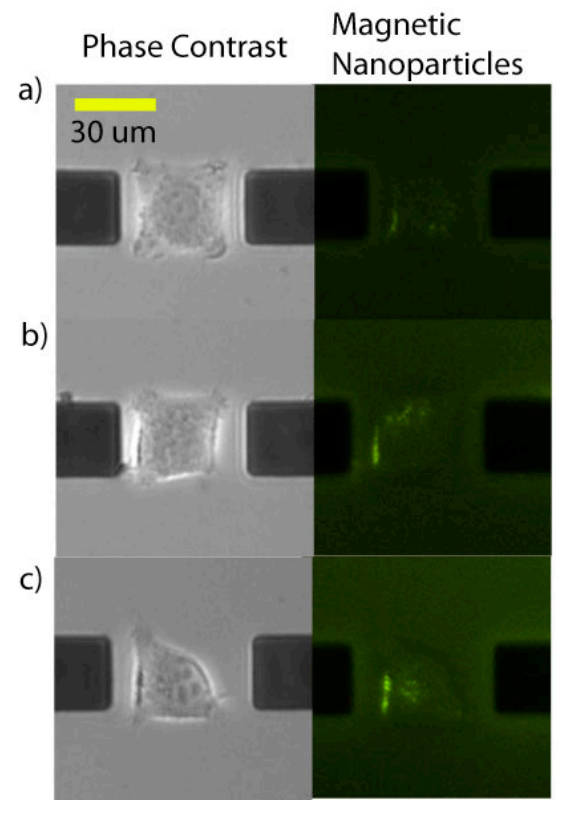

Figure 4: Cells patterned above X fibronectin patterns. Row a) displays the standard cell shape above these particular patterns, as the loaded nanoparticle quantity was low. These cells have characteristically 4 concave sides. Row b) sees a cell with modified shape due to a large density of magnetic nanoparticles, and generates noticeable polarization in cell shape on both the side of the nanoparticles and the other side of the nanoparticles. Row c) sees a cell with even higher nanoparticles, however because these particles are a larger distance from the elements, this yields a lower magnetic field gradient, and thus a lower force. Parameters such as cell distance from element, element size, and fibronectin pattern were all varied on our test chip. 


\section{Results}

Our results indicate the ability to properly shape and control cell localization in close proximity to our magnetic elements. Shown in Figure 4 are merged images of cells, protein patterns, and localized magnetic nanoparticles.

Early results also indicate that the application of forces modifies certain aspects of cell shape stability and polarize the cell shape (Figure 5). Shown in Figure 5 are images of polarized cell structures maintaining their shape over long times, as compared to cells with few to no nanoparticles that commonly destabilize. From these results, we anticipate that these structures can additionally be used to study and influence cell motion.
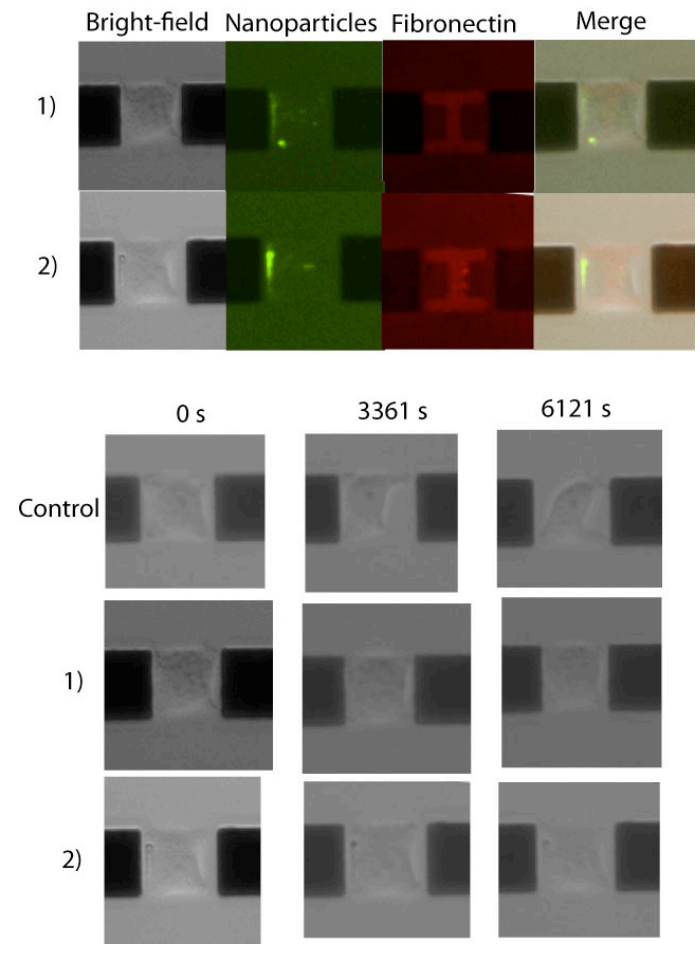

Figure 5: Stability of polarized cell structures under magnetic force influence. Rows 1) and 2) display various channel and merged images of cells strongly polarized by magnetic force. The bottom rows display the differences in stability of these structures against a control structure with no magnetic nanoparticles. The control cells commonly bounced around the pattern, and displayed structural instability. This was not the case of particles under influence of magnetic force, that saw a continuous polarized structure until mitosis.

\section{CONCLUSION}

We have demonstrated precisely aligned, remotely controlled force application on cells within minimal additional perturbation to cellular systems, which should open up avenues in both short term and long term study and actuation of cellular response to chemomechanical signals. The method of force application should allow simple application and deapplication of force, and additionally can track the effect on cells as they are free to move around the top of the substrate.

\section{REFERENCES}

[1] A.J. Maniotis, C.S. Chen, and D.E. Ingber, "Demonstration of mechanical connections between integrins, cytoskeletal filaments, and nucleoplasm that stabilize nuclear structure," Proc Nat Acad Sci USA, 94, 849 (1997).

[2] M. Srboljub, M. Mijailovich, M. Kojic, M. Zivkovic, B. Fabry, and J. J. Fredberg, "A finite element model of cell deformation during magnetic bead twisting," J Appl Physiol 93, 1429 (2002).

[3] M. Zborowski, L. Sun, R. Lee, P. Williams, and J. Chalmers, "Continuous cell separation using novel magnetic quadrupole flow sorter," Journal of Magnetism and Magnetic Materials, 194, 224 (1999).

[4] H. Lee, A.M. Purdon, R.M. Westervelt, "Manipulation of biological cells using a microelectromagnet matrix," Appl. Phys. Lett. 85, 1063 (2004).

[5] C. Ahn, M. Allen, W. Trimmer, Y-N. Jun, and S. Erramilli, “A Fully Integrated Micromachined Magnetic Particle Separator," J. MEMS, 5, 151 (1996).

[6] H. Guillou, A. Depraz-Depland, E. Planus, B. Vianay, J. Chaussy, A. Grinchine, C. Albiges-Rizo, M. R. Block, "Lamellipodia nucleation by filopodia depends on integrin occupancy and downstream Racl signalling," Experimental Cell Research, 314, 478 (2008).

[7] J. Gao, W. Zhang, P. Huang, B. Zhang, X. Zhang, and B.J. Xu, "Intracellular control of fluorescent magnetic nanoparticles," J. Amer. Chem. Soc., 130, 3710 (2009).

[8] A. de Vries, B. Krenn, R. Driel, and J. Kanger, "Micro Magnetic tweezers for nanomanipulation inside live cells," Biophysical Journal, 88, 2137 (2005).

[9] P. Tseng, D. Di Carlo, and J.W. Judy, "Rapid and dynamic intracellular patterning of cell-internalized magnetic fluorescent nanoparticles," Nano Lett., 9 (2009), pp. 30533059. 